\section{(d) \\ CrossMark}

\title{
Sustained inflations and avoiding mechanical ventilation to prevent death or bronchopulmonary dysplasia: a meta-analysis
}

\author{
Hendrik S. Fischer (1) ${ }^{1}$, Georg M. Schmölzer ${ }^{2,3}$, Po-Yin Cheung ${ }^{2,3}$ and \\ Christoph Bührer ${ }^{1}$
}

Affiliations: ${ }^{1}$ Dept of Neonatology, Charité University Medical Centre, Berlin, Germany. ${ }^{2}$ Centre for the Studies of Asphyxia and Resuscitation, Neonatal Research Unit, Royal Alexandra Hospital, Edmonton, AB, Canada. ${ }^{3}$ Dept of Pediatrics, University of Alberta, Edmonton, AB, Canada.

Correspondence: Hendrik S. Fischer, Klinik für Neonatologie, Charité - Universitätsmedizin Berlin, Augustenburger Platz 1, 13353 Berlin, Germany. E-mail: hendrik.fischerdcharite.de

@ERSpublications

Meta-analysis of RCTs in preterm infants showed that delivery room strategies to avoid mechanical ventilation reduced bronchopulmonary dysplasia, whereas sustained inflations had no effect and may even increase mortality http://ow.ly/je6u30mxS58

Cite this article as: Fischer HS, Schmölzer GM, Cheung P-Y, et al. Sustained inflations and avoiding mechanical ventilation to prevent death or bronchopulmonary dysplasia: a meta-analysis. Eur Respir Rev 2018; 27: 180083 [https://doi.org/10.1183/16000617.0083-2018].

ABSTRACT Sustained inflations and avoidance of endotracheal mechanical ventilation (eMV) are delivery room interventions aimed at preventing bronchopulmonary dysplasia (BPD). Their effectiveness is the subject of the present meta-analysis.

The databases MEDLINE, EMBASE and CENTRAL were searched for randomised controlled trials (RCTs) of preterm infants that compared: 1) sustained inflations with intermittent positive-pressure ventilation; and 2) a non-intubated strategy of respiratory support with one that prescribed eMV at an earlier stage. Data extraction and analysis followed the standard methods of the Cochrane Collaboration. The primary outcome was death or $\mathrm{BPD}$, defined as need for oxygen or positive pressure treatment at 36 weeks' postmenstrual age.

Avoiding eMV (nine RCTs, 3486 infants) reduced the risk of death or BPD, with a risk ratio of 0.90 (95\% CI 0.84-0.97) and a number needed to treat of 35. After sustained inflations (six RCTs, 854 infants), the risk ratio was 0.85 (95\% CI 0.65-1.12). A current multicentre RCT of sustained inflations in very preterm infants was halted for increased early mortality in the sustained inflations arm.

While strategies aimed at avoiding eMV had a small but significant impact on preventing BPD, sustained inflations had no effect and may even increase mortality in very preterm infants.

\section{Introduction}

Bronchopulmonary dysplasia (BPD) is a major complication of prematurity. Affected infants are at increased risk of childhood wheeze, exercise-induced bronchoconstriction and long-term respiratory impairment [1]. In addition, BPD has been associated with growth failure, neurodevelopmental impairment, cerebral palsy and poor school-age performance $[2,3]$. According to recent data from the National Institute

\section{This article has supplementary material available from err.ersjournals.com}

Provenance: Submitted article, peer reviewed.

Received: Aug 212018 | Accepted after revision: Oct 232018

Copyright CERS 2018. ERR articles are open access and distributed under the terms of the Creative Commons Attribution Non-Commercial Licence 4.0. 
of Child Health and Human Development (NICHD) neonatal research network, the overall incidence of moderate to severe BPD is $>40 \%$ in preterm infants $<29$ weeks' gestational age and has remained unchanged since 2000 [4]. Consequently, new interventions to prevent BPD are urgently needed, and there is a consensus that appropriate measures should start in the delivery room [2,5]. Sustained inflations and avoiding endotracheal mechanical ventilation (eMV) are promising respiratory interventions to reduce BPD in the delivery room and have been investigated in randomised controlled trials (RCTs) $[2,6]$.

Sustained inflations at the onset of neonatal resuscitation accelerated lung recruitment and achieved a more uniform aeration of proximal and distal gas exchange regions in animal studies [7]. A previous meta-analysis of four RCTs in preterm infants $<33$ weeks' gestational age found that sustained inflations decreased the need for mechanical ventilation [8]. However, neither the aforementioned meta-analysis nor a subsequent Cochrane review showed effects of sustained inflations on BPD $[8,9]$.

Strategies to avoid eMV in preterm infants aim to reduce ventilator-induced lung injury in the delivery room by noninvasive respiratory support and selective surfactant administration. A previous meta-analysis of seven RCTs showed that avoiding eMV by nasal continuous positive airway pressure (nCPAP) alone or by surfactant administration during nCPAP reduced the incidence of death or BPD in preterm infants $<30$ weeks' gestational age [10].

Recently, new RCTs of sustained inflations and of strategies to avoid eMV in the delivery room have been published [11-13]. Therefore, the present meta-analysis aimed to quantitate the effects that sustained inflations and avoiding eMV have on BPD and mortality, considering the most current evidence.

\section{Methods}

The standard methods of the Cochrane Collaboration were applied [14]. For each meta-analysis, the authors re-used the respective study protocol of their previously published meta-analyses about sustained inflations [8] and avoidance of eMV [10], as summarised in the following section. The definite study protocol was registered in the international prospective register of systematic reviews (PROSPERO) [15].

\section{Criteria for considering studies for the meta-analyses}

RCTs published in full in peer-reviewed journals were accepted. No language restrictions were applied. For the meta-analysis about sustained inflations, abstracts from the annual meetings of the Pediatric Academic Societies and the European Society for Paediatric Research from 2000 to 2018 were also accepted.

To examine the effect of sustained inflations, we included RCTs comparing sustained inflations with intermittent positive-pressure ventilation (IPPV) as the primary respiratory intervention in the delivery room in a preterm population of $<33$ weeks' gestational age [8].

To assess the impact of avoiding eMV, we considered RCTs that compared strategies that avoided eMV (e.g. nCPAP) with a control group in which eMV was performed at an earlier stage, e.g. prophylactic mechanical ventilation or INSURE (intubation, surfactant, extubation). Included RCTs had to be published after 2000 and provide data for preterm infants <30 weeks' gestational age. RCTs including more mature infants were eligible if $>50 \%$ of the study patients were $<30$ weeks' gestational age and if the study authors provided stratified data for these infants [10]. Unlike in the previous meta-analysis, we only included studies that prescribed randomisation within the first $2 \mathrm{~h}$ of age, and planned a sensitivity analysis of those studies randomised within the first hour.

\section{Types of outcome measures}

The primary outcome was death or BPD, defined as need for oxygen or positive pressure treatment at 36 weeks' postmenstrual age. To ascertain that benefits in the combined outcome of death or BPD were not associated with increased mortality, death and BPD were also assessed separately as secondary outcomes.

\section{Search methods for identification of studies}

To find RCTs about sustained inflations, two authors (G.M. Schmölzer and P-Y. Cheung) searched MEDLINE, EMBASE and CENTRAL up to July 30, 2018. The search strategy combined the search concepts "infant", "newborn" and "sustained inflation" (see supplementary material for details). In addition, a manual search of the bibliography of all included RCTs was performed, and of conference abstracts, as already described.

To identify RCTs about avoiding eMV, two authors (H.S. Fischer and C. Bührer) searched MEDLINE, EMBASE and CENTRAL from January 2000 up to August 1, 2018, in combination with cross-referencing and occasional access to other resources. The search strategy in the databases combined the search concepts "neonate or prematurity or BPD", "surfactant or ventilatory support" and "randomized controlled trial" (see supplementary material for details). 


\section{Assessment of methodological quality}

In accordance with Cochrane methodology, risk of selection bias, performance bias, detection bias, attrition bias, reporting bias and other risks of bias were assessed, and classified as low, high or unclear risk [14].

\section{Data collection and analysis}

For each meta-analysis, two authors worked independently of each other on selecting the studies, assessing the methodological quality and extracting the data (sustained inflations: G.M. Schmölzer and P-Y. Cheung; avoiding eMV: H.S. Fischer and C. Bührer). Differences were resolved by a third author. RevMan Version 5.3 (Cochrane Collaboration, Nordic Cochrane Centre, Copenhagen, Denmark) was applied to compute intervention effects, using the Mantel-Haenszel method for dichotomous outcomes in a random effects model. Results were reported as risk ratios (RRs) and 95\% confidence intervals (CIs). A p-value of $<0.05$ was considered statistically significant. A number needed to treat (NNT) was calculated for pooled outcomes with significant effects. Chi-squared tests and I-squared values served as measures of heterogeneity. To assess for publication bias, the intervention effects on death or BPD were plotted against the standard error of $\log$ (odds ratio) in a funnel plot [14]. The meta-analyses were reported in accordance with the Preferred Reporting Items for Systematic Reviews and Meta-Analyses (PRISMA) statement [16].

\section{Results}

The selection process of including RCTs in the updated meta-analyses about sustained inflations and avoiding eMV is depicted in figure 1. Overall, the authors screened an additional 3906 records, assessed 35 new full-text articles and included five new RCTs for meta-analysis. Stratified data and additional information were obtained from several study authors (see acknowledgements section).

\section{Excluded studies}

Reasons for exclusion are categorised in figure 1a (sustained inflations) and figure 1b (avoiding eMV). With respect to sustained inflations, most full-text articles were excluded because they did not report RCTs, or investigated other respiratory treatments. With regard to avoiding eMV, full-text articles were excluded mostly because they focused on premature infants $\geqslant 30$ weeks' gestational age, and two RCTs were excluded because they allowed randomisation at $>2 \mathrm{~h}$ of age.

\section{Included studies}

The characteristics of all included RCTs are shown in tables 1 and 2, and are described in detail in the supplementary material. A total of six RCTs about sustained inflations were included. In the sustained

a) Sustained inflations

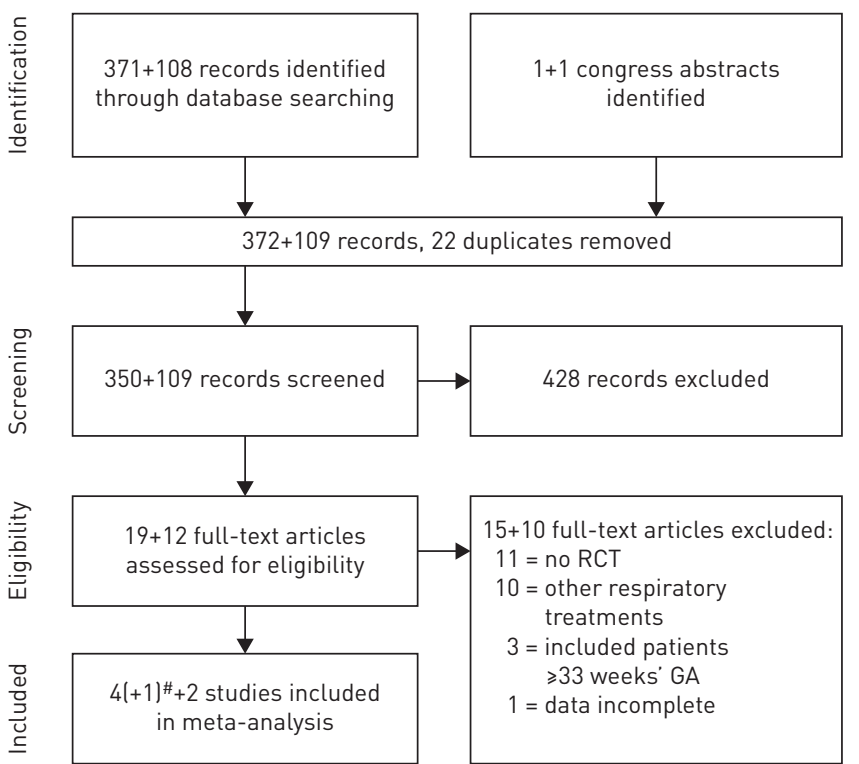

b) Avoiding eMV

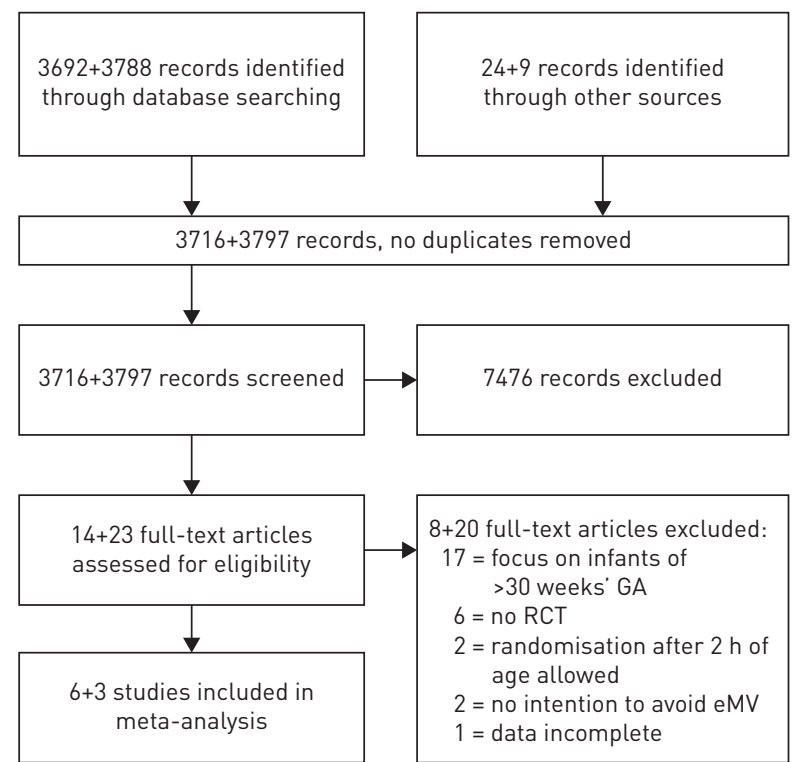

FIGURE 1 Preferred Reporting Items for Systematic Reviews and Meta-Analyses (PRISMA) flow diagrams of the selection of randomised controlled trials (RCTs) about a) sustained inflations and b) avoiding endotracheal mechanical ventilation (eMV). At each stage, the first number refers to the previous version of this meta-analysis, whereas the second number represents the newly identified records. ${ }^{\#}$ : the notation $(+1)$ refers to a letter to the editor, with stratified data about infants <28 weeks' gestational age (GA) from the trial by TE PAs et al. [17]. 
TABLE 1 Characteristics of studies included in the meta-analysis about sustained inflations (SI)

\begin{tabular}{|c|c|c|c|c|c|c|c|c|}
\hline $\begin{array}{l}\text { First author } \\
\text { [ref.] }\end{array}$ & Year & Intervention & $\begin{array}{l}\text { Pressure levels } \# \\
\mathrm{cmH}_{2} \mathrm{O}\end{array}$ & $\begin{array}{l}\text { Ventilation } \\
\text { device }\end{array}$ & $\begin{array}{l}\text { Gestational age } \\
\text { weeks }\end{array}$ & $\begin{array}{l}\text { Antenatal } \\
\text { steroids }\end{array}$ & Patients & Recruitment \\
\hline HaRLing [18] & 2005 & $\begin{array}{c}1 \mathrm{SI} \text { (5 s) versus IPPV } \\
\text { (2 s inflation) }\end{array}$ & $\begin{array}{l}\text { SI: 25-30; } \\
\text { IPPV: } 25-30 / 3-4\end{array}$ & TPR & $\leqslant 306 / 7$ & No data & 52 & Prior to 2005 \\
\hline LINDNER [19] & 2005 & $\begin{array}{l}1-3 \mathrm{SI} \text { (15 s each) } \\
\text { versus IPPV (0.5 s } \\
\quad \text { inflation) }\end{array}$ & $\begin{array}{l}\text { SI: } 20-30 ; \\
\text { IPPV: } 20-30 / 4-6\end{array}$ & $\begin{array}{l}\text { Constant flow } \\
\text { ventilator }\end{array}$ & $25 \%$ to $28 \%$ & $81 \%$ versus $80 \%$ & 61 & 1999-2002 \\
\hline LISTA [20] & 2015 & $\begin{array}{c}1-2 \mathrm{SI} \text { (15 s each) } \\
\text { versus nCPAP } \pm \text { IPPV }\end{array}$ & $\begin{array}{c}\text { SI: 25; } \\
\text { IPPV: PIP 20-25 }\end{array}$ & TPR & $25 \%$ to $28 \% / 7$ & $91 \%$ versus $87 \%$ & 291 & 2011-2013 \\
\hline $\begin{array}{l}\text { JIRAVISITKUL } \\
\text { [11] }\end{array}$ & 2017 & $\begin{array}{c}1-2 \text { SI ( } 15 \text { s each) } \\
\text { versus IPPV }\end{array}$ & $\begin{array}{c}\text { SI: } 25 \\
\text { IPPV: } 15-20 / 5\end{array}$ & TPR & $25 \%$ to $32 \% / 7$ & $63 \%$ versus $74 \%$ & 81 & 2013-2015 \\
\hline
\end{tabular}

IPPV: intermittent positive-pressure ventilation; TPR: T-piece resuscitator; PIP: positive inspiratory pressure; nCPAP: nasal continuous positive airway pressure. ${ }^{\#}$ : during SI or peak inspiratory pressure/positive end-expiratory pressure during IPPV.

inflations arms, 1-3 sustained inflations at a duration of 5-20 s and a pressure of $20-30 \mathrm{cmH}_{2} \mathrm{O}$ were applied (table 1) [11, 12, 17-20]. With regard to avoiding eMV, nine RCTs were included. These RCTs compared various strategies of nCPAP with or without surfactant administration via a thin catheter versus strategies that prescribed INSURE or longer-term eMV and surfactant (table 2) [13, 21-28].

Risk of bias in the included studies

The results of the risk of bias assessment are summarised in figure 2. There was a lack of blinding to the intervention in all RCTs (high risk/performance bias), and to the outcome assessment in most RCTs (high risk/detection bias). Only two RCTs in the sustained inflations meta-analysis prescribed a blinded outcome

TABLE 2 Characteristics of studies included in the meta-analysis about avoiding endotracheal mechanical ventilation (eMV)

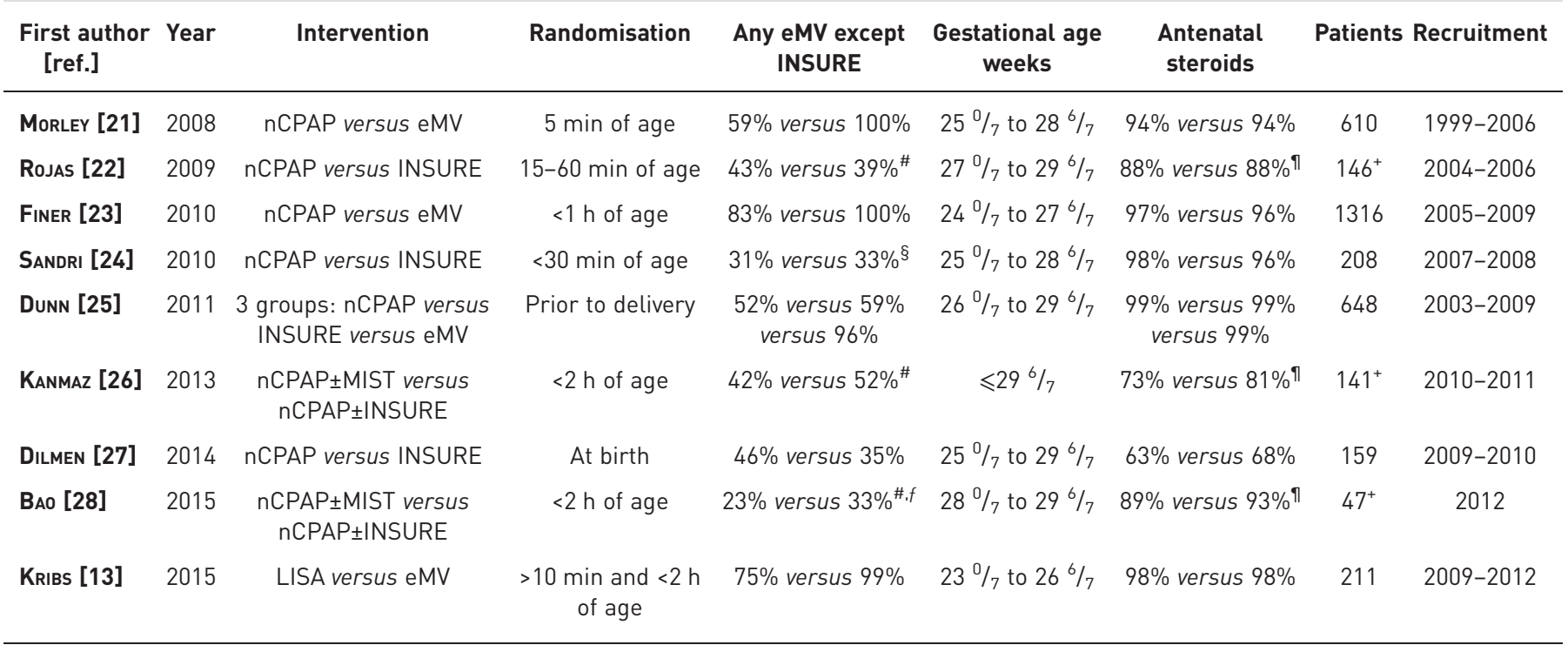

INSURE: intubation, surfactant, extubation; nCPAP: nasal continuous positive airway pressure; MIST: minimally invasive surfactant therapy; LISA: less invasive surfactant administration. ${ }^{\#}$ : stratified data for infants $<30 \%$ weeks' gestational age provided by the study authors; ${ }^{\text {I }}$ : data given for the unstratified dataset; ${ }^{+}$: number of infants $<30 \%$ weeks' gestational age only; ${ }^{\S}$ : need for any eMV except INSURE in the first 5 days of life; ${ }^{f}$ : need for any eMV except INSURE in the first $72 \mathrm{~h}$ of life. 
FIGURE 2 Risk of bias summary: current review authors' judgements about each risk of bias item for each included randomised controlled trial (RCT). +: low risk; -: high risk; ?: unclear risk.

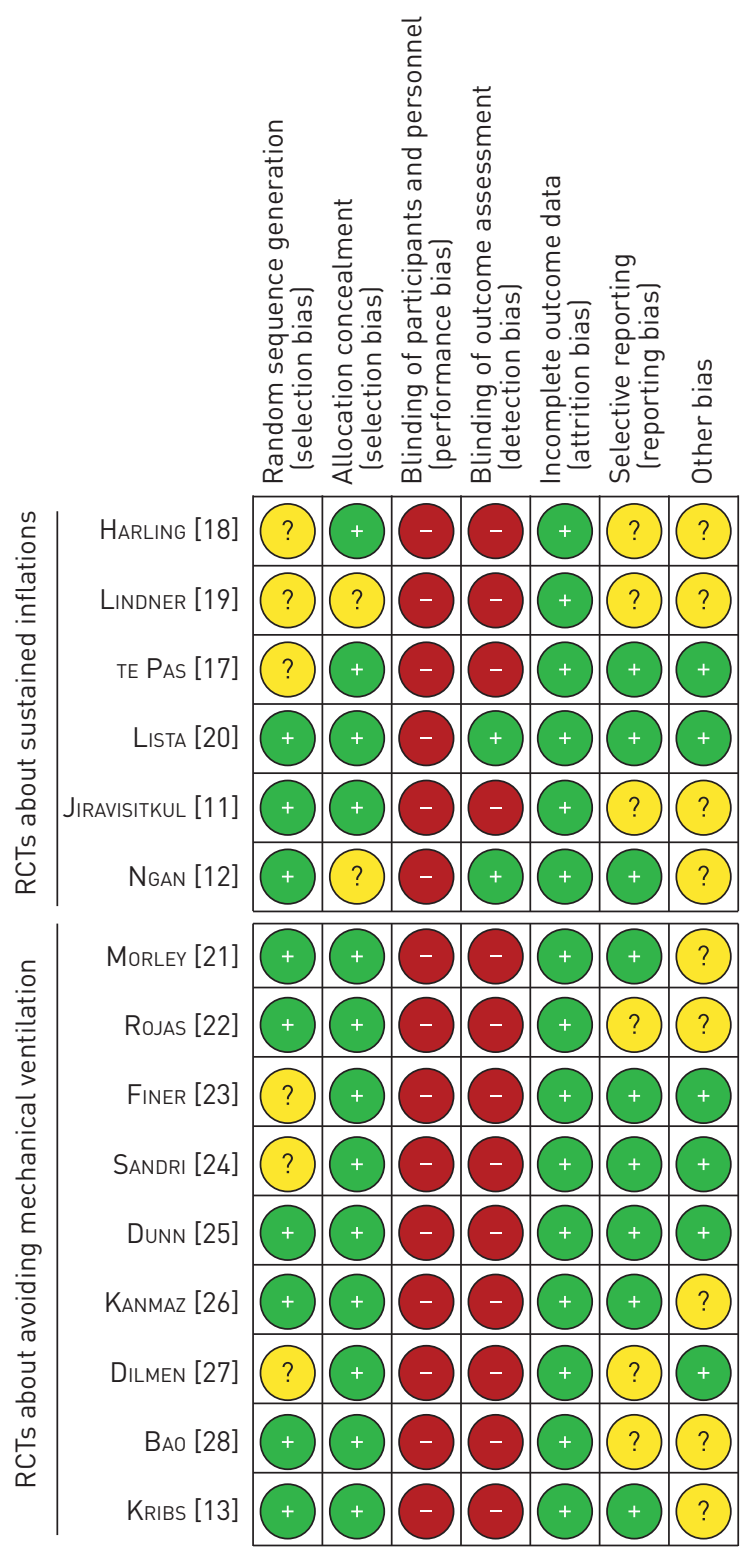

assessment (low risk/detection bias) [12, 20]. Minor issues in several studies were the incomplete description of random sequence generation (unclear risk/selection bias) [17-19, 23, 24, 27], late or no registration of the study protocol (unclear risk/reporting bias) $[11,18,19,22,27,28]$, and the use of respiratory distress as a study entry criterion (unclear risk/sampling bias) [13, 21, 22, 26, 28]. Other risks of bias in individual studies and the support for the current review authors' judgements are described in the supplementary material. Visual inspection of the funnel plots of the effects of sustained inflations and avoiding eMV on death or BPD showed an almost symmetrical distribution of effect sizes around the mean, indicating a low risk of publication bias (supplementary figures S1 and S2).

\section{Effects of intervention}

Sustained inflations had no effect on the combined outcome of death or BPD (RR 0.85, 95\% CI 0.65-1.12; $\mathrm{p}=0.25$; figure $3 \mathrm{a}$ ). When death and BPD were evaluated as separate outcomes, sustained inflations still had no effect on BPD (RR 0.78, 95\% CI 0.57-1.05; $\mathrm{p}=0.10$; figure $3 \mathrm{~b}$ ) and no effect on death (RR 1.31, 95\% CI 0.80-2.14; $\mathrm{p}=0.29$; figure $3 \mathrm{c}$ ).

In the second meta-analysis, the use of various strategies to avoid eMV significantly decreased the risk of death or BPD ( $R R$ 0.90, 95\% CI 0.84-0.97; $\mathrm{p}=0.008$; figure 4a). The NNT was 32. There were no significant effects of avoiding eMV on BPD alone (RR 0.92, 95\% CI 0.83-1.01; p=0.09; figure 4b) or on 


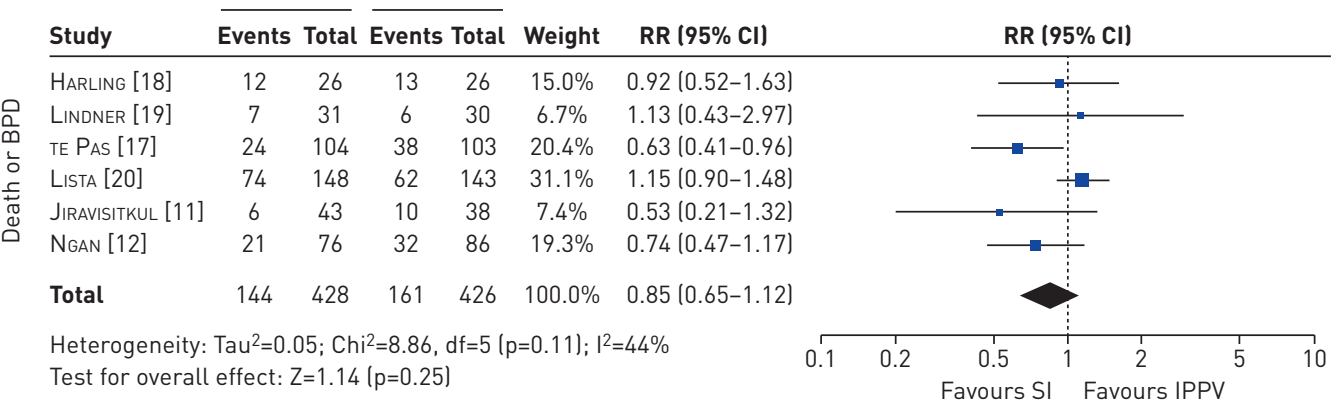

b)

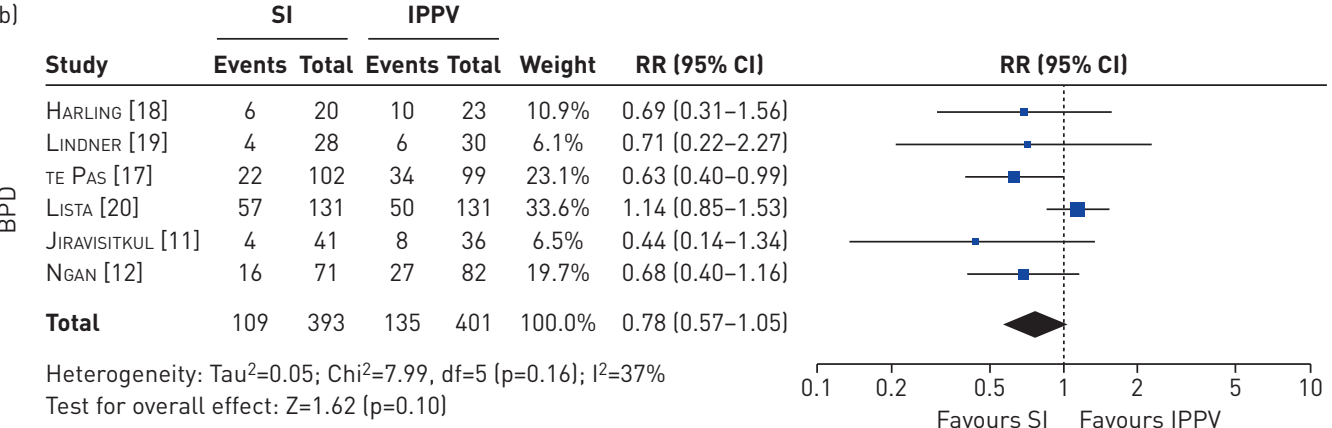

c)

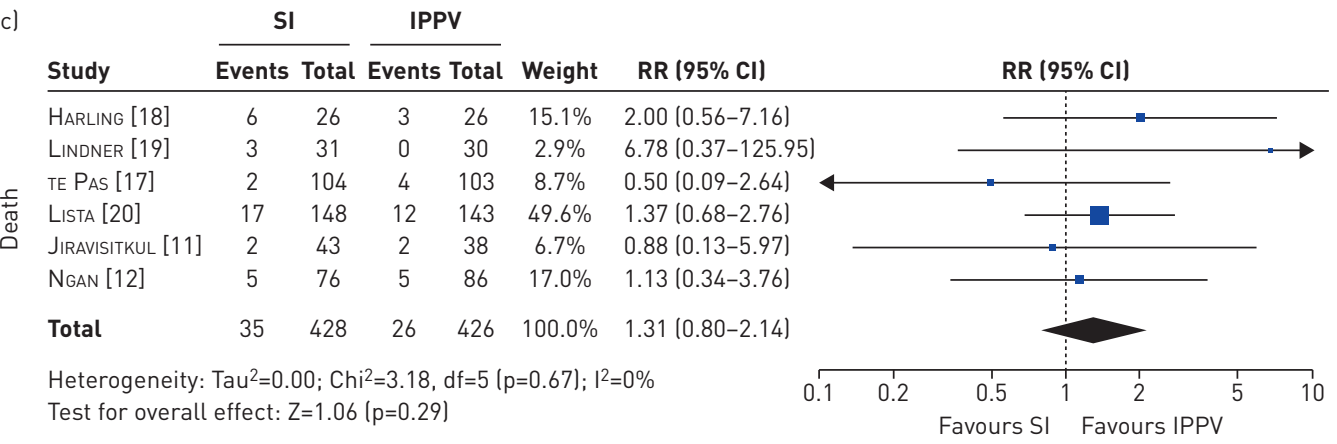

FIGURE 3 Effect of sustained inflations (SI) on a) the combined outcome of death or bronchopulmonary dysplasia (BPD), b) BPD and c) death. Risk ratios (RR) were calculated using the Mantel-Haenszel method for dichotomous outcomes in a random effects model. IPPV: intermittent positive-pressure ventilation.

death (RR 0.88, 95\% CI 0.73-1.06; p=0.18; figure 4c). Sensitivity analyses limited to six studies that prescribed randomisation within the first hour of life confirmed the effect of avoiding eMV on death or BPD (RR 0.91, 95\% CI 0.85-0.99; p=0.03; supplementary figure S3), with an NNT of 44.

Statistical measures indicated some heterogeneity in the meta-analyses of sustained inflations on BPD $\left(\mathrm{I}^{2}=37 \%\right.$; figure $\left.3 \mathrm{~b}\right)$ and of sustained inflations on death or BPD $\left(\mathrm{I}^{2}=44 \%\right.$; figure $\left.3 \mathrm{a}\right)$, while there were no indicators of heterogeneity in the other analyses.

\section{Discussion}

The present meta-analyses showed that sustained inflations had no effect on the combined outcome of death or BPD (figure 3a), whereas delivery room strategies to avoid eMV reduced the risk of death or BPD (figure 4a). The beneficial effect of avoiding eMV was small but significant, with an NNT of 32.

\section{Quality of evidence}

Most studies were of good methodological quality, with low risk of bias in most domains. The only major issue was the lack of blinding (figure 2). Although it is almost impossible to blind clinicians to interventions such as sustained inflations or endotracheal intubation, future RCTs should consider blinding of the outcome assessment, including the use of a blinded, standardised, time-limited room air challenge to assess BPD at 36 weeks' postmenstrual age [10]. Two early RCTs about sustained inflations 
a) Avoid eMV Control group

\begin{tabular}{|c|c|c|c|c|c|c|}
\hline Study & Events & Total & Events & Total & Weight & $\operatorname{RR}(95 \% \mathrm{CI})$ \\
\hline MoRLEy [21] & 108 & 307 & 122 & 303 & $13.4 \%$ & $0.87(0.71-1.07)$ \\
\hline RoJAs [22] & 53 & 74 & 54 & 72 & $14.6 \%$ & $0.95(0.79-1.16)$ \\
\hline FINER [23] & 323 & 663 & 353 & 653 & $50.6 \%$ & $0.90(0.81-1.00)$ \\
\hline SANDRI [24] & 22 & 103 & 23 & 105 & $2.1 \%$ & $0.98(0.58-1.64)$ \\
\hline DunN [25] & 68 & 223 & 138 & 425 & $9.7 \%$ & $0.94(0.74-1.19)$ \\
\hline Kanmaz [26] & 25 & 74 & 30 & 67 & $3.3 \%$ & $0.75(0.50-1.14)$ \\
\hline DILMEN [27] & 16 & 80 & 13 & 79 & $1.3 \%$ & $1.22(0.63-2.36)$ \\
\hline BAO [28] & 7 & 26 & 6 & 21 & $0.7 \%$ & $0.94(0.37-2.38)$ \\
\hline KRIBS [13] & 35 & 107 & 43 & 104 & $4.4 \%$ & $0.79(0.55-1.13)$ \\
\hline Total & 657 & 1657 & 782 & 1829 & $100.0 \%$ & $0.90(0.84-0.97)$ \\
\hline
\end{tabular}

Heterogeneity: $\mathrm{Tau}^{2}=0.00 ; \mathrm{Chi}^{2}=2.64, \mathrm{df}=8(\mathrm{p}=0.95) ; \mathrm{I}^{2}=0 \%$ Test for overall effect: $Z=2.66(p=0.008)$

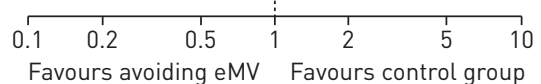

b)

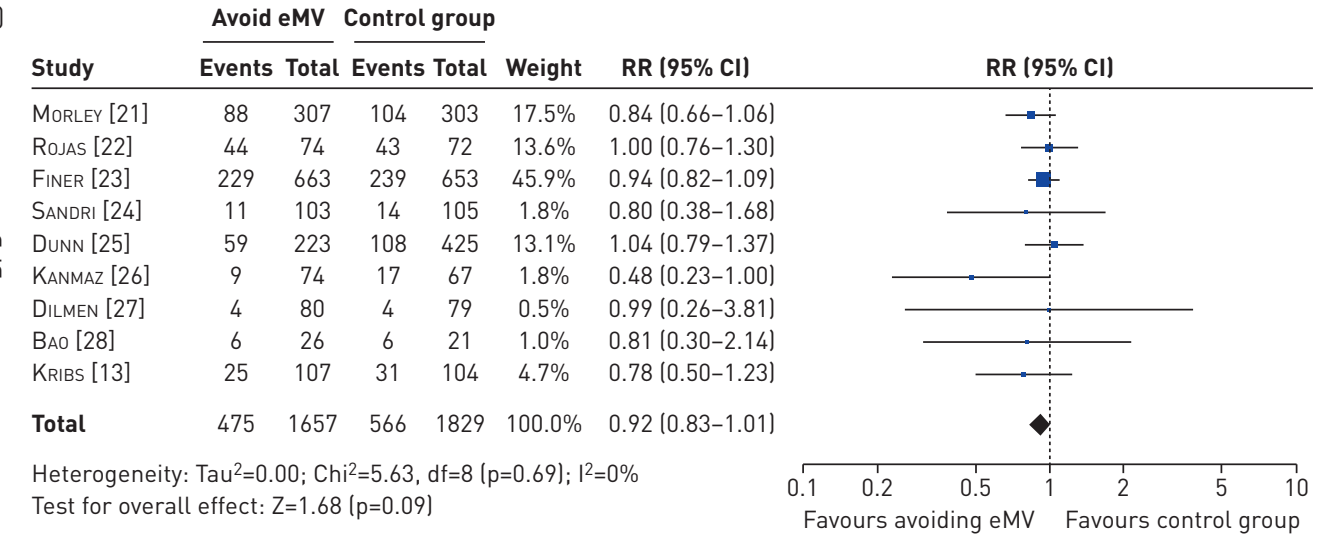

c)

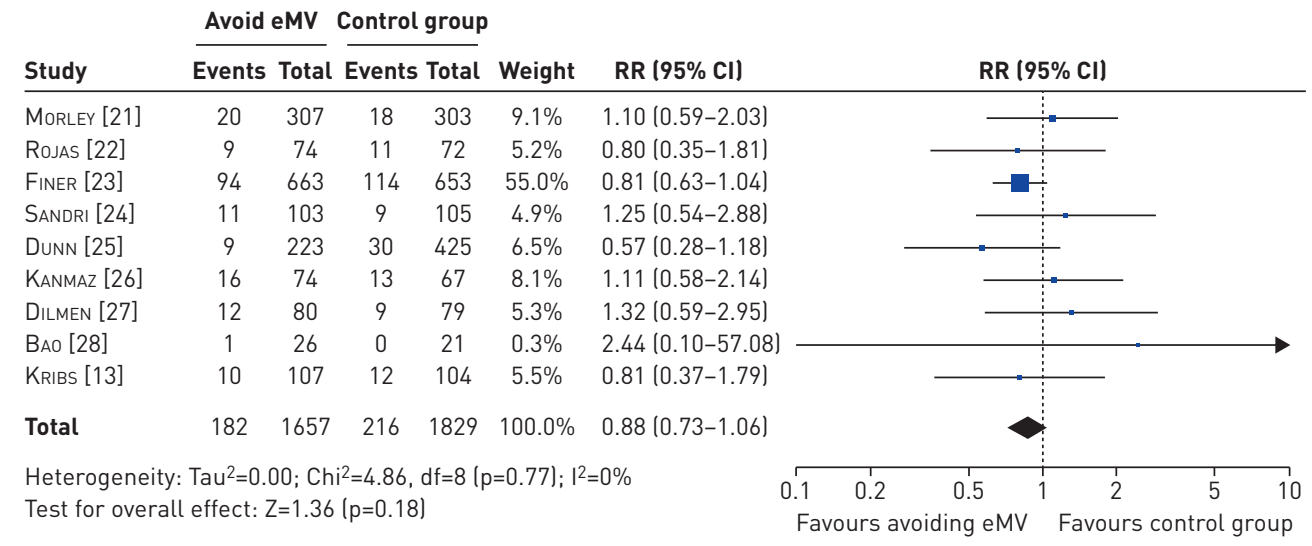

FIGURE 4 Effect of avoiding endotracheal mechanical ventilation (eMV) on a) the combined outcome of death or bronchopulmonary dysplasia (BPD), b) BPD and c) death. Risk ratios (RR) were calculated using the Mantel-Haenszel method for dichotomous outcomes in a random effects model.

were mostly classified as "unclear risk" due to incomplete description of random sequence generation, non-registration of the study protocol and other reasons $[18,19]$, as discussed previously [8].

Effect of sustained inflations on BPD

The meta-analysis of six RCTs including 854 preterm infants of $<33$ weeks' gestational age showed no effects of sustained inflations on BPD, death or the combined outcome of death or BPD (figure 3). This could be due to inefficiency of sustained inflations in infants who remained apnoeic after birth [6], or due to excessive pressure and duration of the sustained inflations, with subsequent inflammatory response in the lungs [29]. Notably, the duration of sustained inflations was guided by exhaled carbon dioxide monitoring in one RCT, but this did not improve outcomes [12]. In any case, the meta-analysis highlights that current protocols of sustained inflations are ineffective to prevent death or BPD in the population studied. 
Although the present meta-analysis did not show an effect of sustained inflations on mortality, preliminary data of the Sustained Aeration of Infant Lungs (SAIL) trial raised concerns about the safety of sustained inflations, as the trial has recently been halted prematurely due to an excess of early deaths ( $<48 \mathrm{~h}$ of age) in the sustained inflations arm (7.5\% versus $1.4 \% ; \mathrm{p}=0.002)$ [30]. SAIL was the largest RCT about sustained inflations to date $(n=425)$ and included immature infants of $\leqslant 26$ weeks' gestational age [31]. The SAIL data could not be meta-analysed by us, because outcomes have only been presented as RR so far. Moreover, the preliminary data by KiRPALANi et al. [30] need to be interpreted with caution, as this meeting abstract is not fully peer-reviewed. Notably, there was a trend towards more BPD (RR 1.12, 95\% CI 0.9-1.4) and higher mortality in the sustained inflations arm (RR 1.33, 95\% CI 0.9-2.0).

Considering the lack of effectiveness of sustained inflations in the present meta-analysis, and the concern about increased mortality in the preliminary data from the SAIL trial, the use of sustained inflations should be strictly limited to research settings. In addition, before new studies about sustained inflations are conducted, data from the SAIL trial and from other RCTs should be thoroughly evaluated for the overall effect of sustained inflations on mortality and on other adverse effects in very immature infants.

\section{Effect of avoiding eMV on BPD}

The second meta-analysis, including nine RCTs with 3486 premature infants of $<30$ weeks' gestational age, showed that avoiding eMV has a role in preventing BPD. Despite a substantial variety in the studied populations and interventions, strategies to avoid eMV had an almost consistent effect on reducing death or BPD, and were not associated with increased mortality. However, the large NNT to avoid one case of death or BPD raises the question of whether specific strategies of nCPAP and surfactant administration are more effective than others.

In premature infants, even brief eMV in the delivery room may have negative effects on the lungs and brain, and may offset the positive impact of surfactant [3]. Accordingly, a meta-analysis of nine RCTs investigating early INSURE versus nCPAP alone failed to show a beneficial effect of INSURE on the outcome of death or BPD [32], and a meta-analysis comparing nCPAP with intubation for INSURE or longer-term eMV in infants <32 weeks' gestational age found less death or BPD in the nCPAP group (RR 0.91, 95\% CI 0.84-0.99; NNT=25) [33]. More recent techniques of "less invasive surfactant administration" (LISA), "minimally invasive surfactant therapy" (MIST) and their modifications apply surfactant during nCPAP [34], aiming to combine the beneficial effects of surfactant with the benefits of a nCPAP-only approach $[13,26,28]$. To date, nCPAP supplemented by LISA/MIST has been the most effective method for reducing death or BPD in meta-analyses, in comparison with various strategies that included brief or longer-term eMV [2, 34-36], or with the INSURE procedure (RR 0.63, 95\% CI 0.44-0.92; NNT=11) [34]. The effect sizes in the above-cited meta-analyses about LISA/MIST must be interpreted with caution, as they included data from infants of up to 32-35 weeks' gestational age [2, 34-36], whereas our meta-analysis only included data from infants of $<30$ weeks' gestational age who have a higher risk of BPD.

In a pre-specified sensitivity analysis limited to studies that prescribed randomisation within the first hour of life, we focused on the RCTs that definitively intervened in the delivery room. Interestingly, this approach excluded all RCTs that applied LISA/MIST $[13,26,28]$, and further reduced the benefit of avoiding eMV (supplementary figure S3). This effect could be due to differences in the study protocols and populations studied, but would also be consistent with a higher effectiveness of strategies that apply LISA/MIST.

Overall, the beneficial effect of existing strategies to avoid eMV appears to be limited, which is disappointing from a clinical point of view.

\section{Can the avoidance of eMV be optimised to better prevent BPD?}

To date, more innovative ways of surfactant administration, such as pharyngeal instillation before the first breath, aerosolisation or application via a laryngeal mask, have only been tested in feasibility studies or pilot trials [37]. A Cochrane review showed that early nasal IPPV within the first $6 \mathrm{~h}$ of life avoided eMV more effectively than nCPAP, but only a subgroup analysis limited to four RCTs that applied synchronised nasal IPPV indicated a trend towards less BPD (RR 0.43, 95\% CI 0.18-1.01; p=0.053) [38]. Nasal highfrequency oscillatory ventilation is a promising alternative to nasal IPPV in preterm infants $<1500 \mathrm{~g}$ [39], but to date, no RCT has investigated its use prior to endotracheal intubation. Finally, clinical studies showed that high-flow nasal cannula was equivalent or inferior to nCPAP in preventing eMV and did not have an impact on BPD [40]. Although more data of ongoing trials are currently awaited, it is unlikely that new strategies to avoid eMV in the delivery room will lead to major improvements in BPD prevention in the near future. 
clinical strategies should integrate established delivery room interventions with moderate to marginal effect size to achieve a better outcome. Neonatologists should follow current guidelines and recommendations, including the use of nCPAP to avoid $\mathrm{eMV}$ in the delivery room, and early rescue surfactant administration, ideally by LISA or MIST $[2,5,41]$. Innovative interventions should be further investigated in RCTs, e.g. caffeine administration in the delivery room and admixture of budesonide to intratracheal surfactant instillation [2]. At present, however, sustained inflations cannot be recommended to prevent $\mathrm{BPD}$ in preterm infants.

\section{Limitations of the meta-analyses}

As the scope of the present meta-analyses was limited to the outcomes of death and/or BPD, more comprehensive meta-analyses are needed to investigate the impact of sustained inflations and of specific strategies to avoid eMV on other adverse outcomes of prematurity. Moreover, in the meta-analysis of avoiding eMV, included RCTs that applied LISA/MIST allowed intervention within $2 \mathrm{~h}$ after birth, sometimes after transfer to the neonatal unit, thereby diverging from our focus on delivery room management. The RCTs included in the sensitivity analysis, however, all prescribed intervention in the delivery room (supplementary figure S3). Although statistical measures indicated only modest heterogeneity in two analyses (figures $3 \mathrm{a}$ and b), methodological diversity between trials may have had an impact on the meta-analysis results [14], as the meta-analysed RCTs differed with regard to inclusion criteria, interventions and treatment protocol. In particular, different gestational ages of the study patients and different respiratory care strategies are apparent in the trials included (tables 1 and 2). Moreover, the outcome definitions of BPD also differed between trials: while seven RCTs defined moderate to severe BPD as the need for oxygen treatment at 36 weeks' postmenstrual age, six RCTs applied the NICHD workshop consensus definition [42] and two RCTs used the physiological definition [43].

\section{Conclusions}

Prevention of BPD starts in the delivery room and requires a comprehensive strategy. The present meta-analysis of sustained inflations and preliminary data from the SAIL trial indicate that current protocols of sustained inflations do not prevent BPD and may even increase mortality in very preterm infants. The use of sustained inflations should therefore be limited to research settings. In contrast, the second meta-analysis showed that avoiding eMV in the delivery room by using nCPAP with selective surfactant administration reduced the combined outcome of death or BPD, and should thus be applied in the clinical setting. However, as only one additional infant survived to 36 weeks' postmenstrual age without BPD for every 32 patients treated in this way, more effective interventions to prevent BPD are urgently needed.

Acknowledgements: The authors would like to thank Jiajun Zhu (Zhejiang University, Hangzhou, China), H. Gözde Kanmaz (Zekai Tahir Burak Maternity Teaching Hospital, Ankara, Turkey) and Mario A. Rojas (Valley Children's Hospital, Madera, CA, USA), for providing previously unpublished data and information on their RCTs. Moreover, we thank Hatice T. Aksoy (Zekai Tahir Burak Maternity Teaching Hospital), Giovanna Bertini (Careggi University Hospital, Florence, Italy), Mohammad Heidarzadeh (Tabriz University, Tabriz, Iran), Kayvan Mirnia (Tabriz University), Maryam Nakhshab (Mazandaran University, Sari, Iran) and Lian Zhang (Guangzhou Women and Children's Medical Center, Guangzhou, China), for providing additional information about their studies, and the public for donating money to the Heart and Stroke Foundation of Canada.

Author contributions: Conception: C. Bührer. Design of the respective meta-analyses, data acquisition and risk of bias assessment: H.S. Fischer, C. Bührer, G.M. Schmölzer and P-Y. Cheung. Data analysis and drafting of the manuscript: H.S. Fischer. All authors critically reviewed and revised the manuscript, and approved the final version as submitted.

Conflict of interest: H.S. Fischer has nothing to disclose. G.M. Schmölzer has nothing to disclose. P-Y. Cheung has nothing to disclose. C. Bührer reports personal fees from Chiesi Pharmaceuticals (manufacturer of surfacant), outside the submitted work.

Support statement: Georg M. Schmölzer is a recipient of the Heart and Stroke Foundation/University of Alberta Professorship of Neonatal Resuscitation, a National New Investigator of the Heart and Stroke Foundation Canada and an Alberta New Investigator of the Heart and Stroke Foundation Alberta. Funding information for this article has been deposited with the Crossref Funder Registry.

\section{References}

1 Cousins M, Hart K, Gallacher D, et al. Long-term respiratory outcomes following preterm birth. Rev Med Clin Condes 2018; 29: 87-97.

2 Foglia EE, Jensen EA, Kirpalani H. Delivery room interventions to prevent bronchopulmonary dysplasia in extremely preterm infants. J Perinatol 2017; 37: 1171-1179.

3 Barton SK, Tolcos M, Miller SL, et al. Unraveling the links between the initiation of ventilation and brain injury in preterm infants. Front Pediatr 2015; 3: 97.

4 Stoll BJ, Hansen NI, Bell EF, et al. Trends in care practices, morbidity, and mortality of extremely preterm neonates, 1993-2012. JAMA 2015; 314: 1039-1051. 
5 Wyllie J, Perlman JM, Kattwinkel J, et al. Part 7: Neonatal resuscitation: 2015 International Consensus on Cardiopulmonary Resuscitation and Emergency Cardiovascular Care Science with Treatment Recommendations. Resuscitation 2015; 95: e169-e201.

6 Lista G, La Verde PA, Castoldi F. Sustained inflation and its role in the delivery room management of preterm infants. Neonatology 2016; 109: 366-368.

7 te Pas $A B$, Siew M, Wallace MJ, et al. Establishing functional residual capacity at birth: the effect of sustained inflation and positive end-expiratory pressure in a preterm rabbit model. Pediatr Res 2009; 65: 537-541.

8 Schmölzer GM, Kumar M, Aziz K, et al. Sustained inflation versus positive pressure ventilation at birth: a systematic review and meta-analysis. Arch Dis Child Fetal Neonatal Ed 2015; 100: F361-F368.

9 Bruschettini M, O'Donnell CP, Davis PG, et al. Sustained versus standard inflations during neonatal resuscitation to prevent mortality and improve respiratory outcomes. Cochrane Database Syst Rev 2017; 7: CD004953.

10 Fischer HS, Bührer C. Avoiding endotracheal ventilation to prevent bronchopulmonary dysplasia: a meta-analysis. Pediatrics 2013; 132: e1351-e1360.

11 Jiravisitkul P, Rattanasiri S, Nuntnarumit P. Randomised controlled trial of sustained lung inflation for resuscitation of preterm infants in the delivery room. Resuscitation 2017; 111: 68-73.

12 Ngan AY, Cheung PY, Hudson-Mason A, et al. Using exhaled $\mathrm{CO}_{2}$ to guide initial respiratory support at birth: a randomised controlled trial. Arch Dis Child Fetal Neonatal Ed 2017; 102: F525-F531.

13 Kribs A, Roll C, Göpel W, et al. Nonintubated surfactant application vs conventional therapy in extremely preterm infants: a randomized clinical trial. JAMA Pediatr 2015; 169: 723-730.

14 Higgins JPT, Green S, eds. Cochrane Handbook for Systematic Reviews of Interventions. Version 5.1.0 (updated March 2011). London, The Cochrane Collaboration, 2011. Available from: www.handbook.cochrane.org

15 Fischer H, Schmölzer GM, Cheung PY, et al. Sustained inflations and avoidance of endotracheal mechanical ventilation as delivery room interventions to prevent bronchopulmonary dysplasia: a meta-analysis. PROSPERO 2018 CRD42018102762. Available from: www.crd.york.ac.uk/PROSPERO/display_record.php?ID=CRD42018102762

16 Liberati A, Altman DG, Tetzlaff J, et al. The PRISMA statement for reporting systematic reviews and meta-analyses of studies that evaluate healthcare interventions: explanation and elaboration. BMJ 2009; 339: b2700.

17 te Pas $\mathrm{AB}$, Walther FJ. A randomized, controlled trial of delivery-room respiratory management in very preterm infants. Pediatrics 2007; 120: 322-329.

18 Harling AE, Beresford MW, Vince GS, et al. Does sustained lung inflation at resuscitation reduce lung injury in the preterm infant? Arch Dis Child Fetal Neonatal Ed 2005; 90: F406-F410.

19 Lindner W, Högel J, Pohlandt F. Sustained pressure-controlled inflation or intermittent mandatory ventilation in preterm infants in the delivery room? A randomized, controlled trial on initial respiratory support via nasopharyngeal tube. Acta Paediatr 2005; 94: 303-309.

20 Lista G, Boni L, Scopesi F, et al. Sustained lung inflation at birth for preterm infants: a randomized clinical trial. Pediatrics 2015; 135: e457-e464.

21 Morley CJ, Davis PG, Doyle LW, et al. Nasal CPAP or intubation at birth for very preterm infants. N Engl J Med 2008; 358: 700-708.

22 Rojas MA, Lozano JM, Rojas MX, et al. Very early surfactant without mandatory ventilation in premature infants treated with early continuous positive airway pressure: a randomized, controlled trial. Pediatrics 2009; 123: $137-142$.

23 Finer NN, Carlo WA, Walsh MC, et al. Early CPAP versus surfactant in extremely preterm infants. SUPPORT Study Group of the Eunice Kennedy Shriver NICHD Neonatal Research Network. N Engl J Med 2010; 362: 1970-1979.

24 Sandri F, Plavka R, Ancora G, et al. Prophylactic or early selective surfactant combined with nCPAP in very preterm infants. Pediatrics 2010; 125: e1402-e1409.

25 Dunn MS, Kaempf J, de Klerk A, et al. Randomized trial comparing 3 approaches to the initial respiratory management of preterm neonates. Pediatrics 2011; 128: e1069-e1076.

26 Kanmaz HG, Erdeve O, Canpolat FE, et al. Surfactant administration via thin catheter during spontaneous breathing: randomized controlled trial. Pediatrics 2013; 131: e502-e509.

27 Dilmen U, Özdemir R, Tatar Aksoy H, et al. Early regular versus late selective poractant treatment in preterm infants born between 25 and 30 gestational weeks: a prospective randomized multicenter study. J Matern Fetal Neonatal Med 2014; 27: 411-415.

28 Bao Y, Zhang G, Wu M, et al. A pilot study of less invasive surfactant administration in very preterm infants in a Chinese tertiary center. BMC Pediatr 2015; 15: 21.

29 Abd El-Fattah N, Nasef N, Al-Harrass MF, et al. Sustained lung inflation at birth for preterm infants at risk of respiratory distress syndrome: the proper pressure and duration. J Neonatal Perinatal Med 2017; 10: 409-417.

30 Kirpalani H, Ratcliffe S, Keszler M, et al. The international "Sustained Aeration for Infant Lung" (SAIL) randomized trial. Presented at the Pediatric Academic Societies meeting, May 5-8, 2018, Toronto. Abstract 1852.1.

31 Foglia EE, Owen LS, Thio M, et al. Sustained Aeration of Infant Lungs (SAIL) trial: study protocol for a randomized controlled trial. Trials 2015; 16: 95

32 Isayama T, Chai-Adisaksopha $\mathrm{C}$, McDonald SD. Noninvasive ventilation with $v s$ without early surfactant to prevent chronic lung disease in preterm infants: a systematic review and meta-analysis. JAMA Pediatr 2015; 169: $731-739$

33 Schmölzer GM, Kumar M, Pichler G, et al. Non-invasive versus invasive respiratory support in preterm infants at birth: systematic review and meta-analysis. BMJ 2013; 347: f5980.

34 Rigo V, Lefebvre C, Broux I. Surfactant instillation in spontaneously breathing preterm infants: a systematic review and meta-analysis. Eur J Pediatr 2016; 175: 1933-1942.

35 Aldana-Aguirre JC, Pinto M, Featherstone RM, et al. Less invasive surfactant administration versus intubation for surfactant delivery in preterm infants with respiratory distress syndrome: a systematic review and meta-analysis. Arch Dis Child Fetal Neonatal Ed 2017; 102: F17-F23.

36 Isayama $\mathrm{T}$, Iwami $\mathrm{H}, \mathrm{McD}$ onald $\mathrm{S}$, et al. Association of noninvasive ventilation strategies with mortality and bronchopulmonary dysplasia among preterm infants: a systematic review and meta-analysis. JAMA 2016; 316 : $611-624$. 
37 Roberts KD, Brown R, Lampland AL, et al. Laryngeal mask airway for surfactant administration in neonates: a randomized, controlled trial. J Pediatr 2018; 193: 40-46.e1.

38 Lemyre B, Laughon M, Bose C, et al. Early nasal intermittent positive pressure ventilation (NIPPV) versus early nasal continuous positive airway pressure (NCPAP) for preterm infants. Cochrane Database Syst Rev 2016; 12: CD005384.

39 Fischer HS, Bohlin K, Bührer C, et al. Nasal high-frequency oscillation ventilation in neonates: a survey in five European countries. Eur J Pediatr 2015; 174: 465-471.

40 Roehr CC, Yoder BA, Davis PG, et al. Evidence support and guidelines for using heated, humidified, high-flow nasal cannulae in neonatology: Oxford Nasal High-Flow Therapy Meeting, 2015. Clin Perinatol 2016; 43: 693-705.

41 Sweet DG, Carnielli V, Greisen G, et al. European Consensus Guidelines on the Management of Respiratory Distress Syndrome - 2016 Update. Neonatology 2017; 111: 107-125.

42 Jobe AH, Bancalari E. Bronchopulmonary dysplasia. Am J Respir Crit Care Med 2001; 163: 1723-1729.

43 Walsh MC, Wilson-Costello D, Zadell A, et al. Safety, reliability, and validity of a physiologic definition of bronchopulmonary dysplasia. J Perinatol 2003; 23: 451-456. 